\title{
Nanotechnology-Based Ozone Sensors Exploiting Low- Frequency Impedance Changes for Detection
}

\author{
Daniele Ziegler ${ }^{1}$, Elena Bekyarova², Gautam Naishadham³ ${ }^{3}$ Patrizia Savi ${ }^{4}$,Andrea Marchisio ${ }^{1}$, \\ , Jean-Marc Tulliani ${ }^{1}$, Krishna Naishadham ${ }^{3}$ \\ 1 Department of Applied Science and Technology, Politecnico di Torino, 10129, Torino, Italy \\ 2 Carbon Solutions Inc., 1200 Columbia Ave, Riverside, CA 92507, USA \\ 3 Wi-Sense LLC, Georgia Tech Campus, Atlanta GA 30332, USA \\ ${ }^{4}$ Department of Electronics and Telecommunications, Politecnico di Torino, 10129, Torino, Italy \\ Daniele Ziegler: daniele.ziegler@polito.it
}

\begin{abstract}
Scientific studies on pollutants and their health effects indicate that real-time monitoring of environmental triggers can improve public health and reduce healthcare costs. This paper introduces sensitive detectors based on functionalized single-walled carbon nanotubes (CNTs) to monitor ozone concentration at sub-ppm level. The CNTs were dispersed and sonicated into an ink formulation which was spray-coated onto interdigitated electrodes on a low-cost commercial substrate. The complex impedance of the sensor signal is measured in the laboratory at frequency between $1 \mathrm{kHz}$ and $200 \mathrm{kHz}$ using a benchtop Impedance Meter. Shunt conductance and capacitance extracted from these measurements on several ozone sensors reveal sensitivity of over $60 \%$ at room temperature below $50 \mathrm{kHz}$, and sensor retractability during desorption.
\end{abstract}

Keywords: carbon nanotubes, environmental pollution, functionalization, gas sensor, ozone exposure

\section{Introduction}

Traffic emissions and industrial pollution release harmful levels of ozone $\left(\mathrm{O}_{3}\right)$, nitrogen dioxide $\left(\mathrm{NO}_{2}\right)$, volatile organic compounds (VOCs) and ultrafine particulate matter (PM) into ambient air, resulting in higher incidence of morbidity and mortality in respiratory diseases [1]. Historically, pollution triggers such as ozone and PM have been integrated into a time-averaged regional air quality index (AQI) measured by field equivalent monitors of regulatory agencies, such as the Environmental Protection Agency (EPA) in the US. People gather pollution data from $A Q$ l reported in the media and make decisions potentially affecting their personal health. However, the monitors may be located tens of $\mathrm{km}$ away from a subject. Hence, the AQI does not provide information pertinent to the patient's microenvironment, typically the breathing zone within a meter around the subject. It appears crucial to characterize the chemical exposure in this microenvironment to mitigate the effects of spatial gradients inherent in AQI data [2]. Additionally, sample integration over time may miss transient high-level exposures. Scientific studies emphasize the need for exposure monitoring in real-time using mobile or portable sensors, which require low-power operation at room temperature without heaters.

This study proposes sensitive detectors based on functionalized single-walled CNTs to measure ozone at sub-ppm level. CNT-based sensors offer sensitivity and compactness, providing a versatile platform to develop breathing zone personal exposure monitoring systems operating in ambient environment. The unique electronic properties of CNTs were used together with the tremendous potential of chemistry for modulating their molecular sensitivity. Tailored chemical functionalization of CNTs (e.g., by polymerization) dramatically improves the adsorption of specific molecules, which in turn affects the electronic properties of the nanotubes, thereby enhancing the sensor's ability for low-power integration with electronic circuitry for signal conditioning and data processing.

\section{Scientific Methods}

The proposed ozone sensors utilize functionalized CNTs to prepare thin-films as gas-sensitive elements. While pristine CNTs constitute a valuable platform for the development of sensitive gas detectors, the chemical modification of their structure offers unique opportunities for improving the sensor performance. In this work, pristine CNTs are functionalized by covalent modification with octadecylamine (ODA) groups for ozone detection [3]. The nanomaterials are dispersed in a compatible solvent (Tetrahydrofuran, THF) to prepare the ink for film deposition, using ultrasonication to break the agglomerates. The CNT thin-film is deposited on interdigitated copper electrodes using commercialgrade air-brush spray coating apparatus. The electrodes are separately fabricated by photolithography on $0.79 \mathrm{~mm}$ thick FR-4 substrate (dielectric constant of 4.1), yielding an interdigitated capacitor (IDC) with 42 fingers, each of length $20 \mathrm{~mm}$ and width $0.2 \mathrm{~mm}$. The IDC has an overall size of $25 \times 25 \mathrm{~mm}^{2}$ and a measured capacitance of $25 \mathrm{pF}$. The DC resistance of the device is measured in-situ 
during CNT deposition to assure film formation with a desired thickness and homogeneity.

\section{Results and Discussion}

Ozone sensors with ODA functionalization were tested in a controlled gas flow system using highprecision ozone generator. The UV lamp (SOG-01, UVP-LLC Cambridge, UK) steps through concentration levels from $200 \mathrm{ppb}$ up to $500 \mathrm{ppb}$. The impedance (magnitude and phase) was measured at frequencies between $1 \mathrm{kHz}$ to $200 \mathrm{kHz}$ using Hioki IM3533 LCR Meter. The IDC sensor is represented by a parallel RC circuit, with the resistance $R$ inversely proportional to the surface conductivity of the film and the capacitance change $\Delta C$ related to the series macro-capacitance between nanotubes. Both these parameters are extracted from the magnitude and phase of the measured impedance. As a representative example, Fig. 1 plots frequency dependence of the measured capacitance for ODA sensor S39, over one cycle of ozone exposure at $200 \mathrm{ppb}$. The time duration of ozone absorption (upswing) and desorption (down swing) cycles as well as the amplitude range are different for each frequency, resulting in unequal total observation times. While the ozone exposure at each frequency is equilibrated till the peak reaches a plateau, reversibility is slow and was not equilibrated. This feature will be corrected by adjusting the CNT networks to optimize the film.

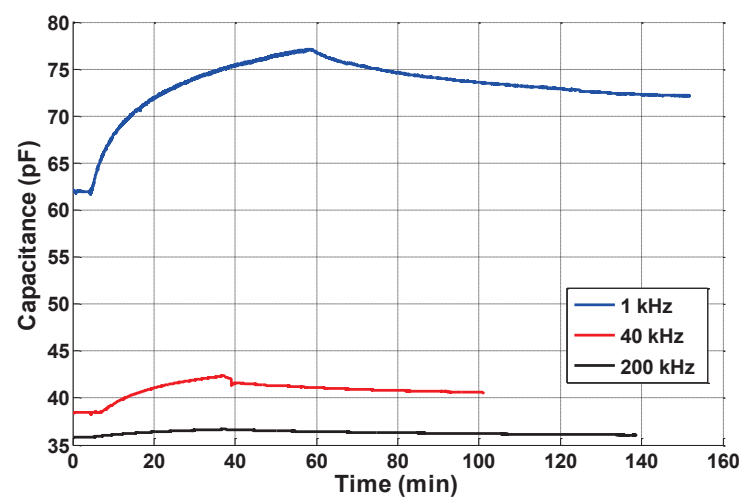

Fig. 1. Frequency dependence of the capacitance extracted from the complex impedance of ODA S39.

Table 1 displays the sensors's response in conductance $G\left(\Delta G / G_{0}\right)$ and capacitance $C\left(\Delta C / C_{0}\right)$ of sensor $\mathrm{S} 39$ extracted from impedance measurements over 1 to $200 \mathrm{kHz}$ range. It is seen

SEM image

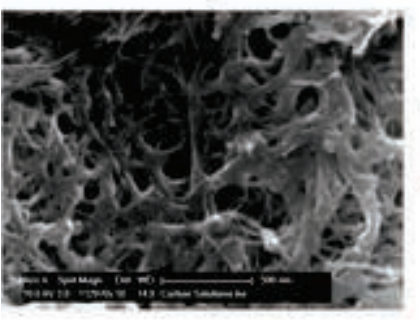

Thermogravimetric Analysis

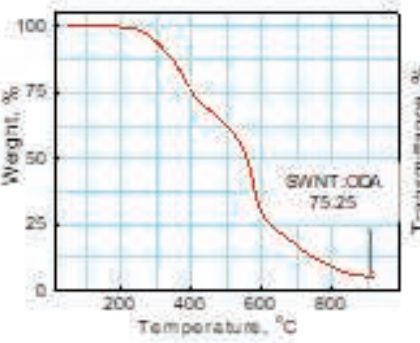

that $G$ offers the maximum response. It is strongly emphasized that $G$ is calculated using both magnitude and phase of the impedance, and therefore cannot be obtained from a purely chemiresistive DC measurement.

Tab. 1: $\quad$ S39 sensor's response.

\begin{tabular}{|c|c|c|}
\hline $\begin{array}{c}\text { Freq. } \\
(\mathrm{kHz})\end{array}$ & $\begin{array}{c}\text { Response in } \\
\text { conductance (\%) }\end{array}$ & $\begin{array}{c}\text { Response in } \\
\text { capacitance (\%) }\end{array}$ \\
\hline 1 & 67.38 & 24.19 \\
\hline 40 & 61.39 & 9.80 \\
\hline 200 & 23.74 & 2.51 \\
\hline
\end{tabular}

The functionalized nanomaterial is characterized by SEM (morphology), TGA (mass losses), mid-IR spectroscopy (proof of covalent functionalization) and UV-Vis-NIR spectroscopy (electronic structure of SWNTs); representative data are shown in Fig. 2. The morphology of the ODA film shows the formation of a highly porous network in the fibrous CNT bundles. The TGA analysis of the components indicates an optimal ODA:CNT weight ratio of 1:3, and the NIR-IR data verifies the existence of oxygencontaining functional groups. Upon functionalization with alkylamines, the peaks around $2922-2853 \mathrm{~cm}^{-1}$ appeared, corresponding to the $\mathrm{C}-\mathrm{H}$ stretching vibration of the alkyl chain $\left(\mathrm{CH}_{2}\right)$. $\mathrm{C}-\mathrm{H}$ bond signal is shifted to 1463 and typical $\mathrm{C}=\mathrm{O}$ band at $1651 \mathrm{~cm}^{-1}$ is present.

\section{References}

[1] B. Brunekreef, S. T. Holgate, "Air pollution and health,"Review Article in The Lancet 360, 12331242 (2002). doi 10.1016/S0140-6736(02)112748.

[2] L.J.S Liu., M. Box, D. Kalman, J. Kaufman, et al., "Exposure assessment of particulate matter for susceptible populations in Seattle," Environ. Health Perspect. 111, 909-918 (2003). doi10.1289/ehp.6011.

[3] E. Bekyarova, M. E. Itkis, N. Cabrera, B. Zhao, A. Yu, R. C. Haddon, Electronic properties of singlewalled carbon nanotube networks. J. Am. Chem. Soc. 127, 5990-5995 (2005). Doi 10.1021/ja043153I
Mid IR Spectroscopy

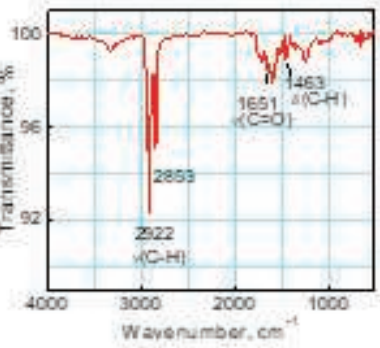

Near Infrared Spectroscopy

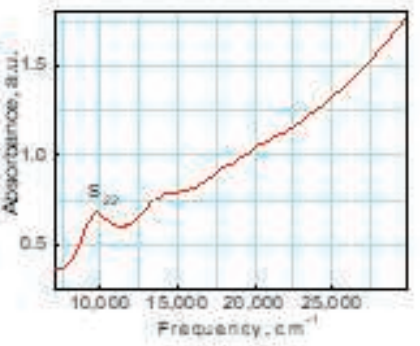

Fig.2. Characterization data for ODA-functionalized CNTs: (a) SEM image showing the porous nanotube networks, (b) TGA analysis for the estimation of ODA loading, (c) Mid IR and (d) Near IR Spectroscopy. 